\title{
Blocking PI3K/Akt signaling attenuates metastasis of nasopharyngeal carcinoma cells through induction of mesenchymal-epithelial reverting transition
}

\author{
HANGUO JIANG, MEI GAO, ZHIHUA SHEN, BOTAO LUO, RUJIA LI, XIAOFAN JIANG, \\ RANRAN DING, YANPING HA, ZHENLIANG WANG and WEI JIE \\ Department of Pathology, School of Basic Medicine Science, Guangdong Medical College, \\ Zhanjiang, Guangdong 524023, P.R. China
}

Received February 12, 2014; Accepted April 9, 2014

DOI: $10.3892 /$ or.2014.3220

\begin{abstract}
In the present study, we evaluated the role of phosphatidylinositol-3 OH kinase/protein kinase B (PI3K/Akt) signaling on changes to epithelial-to-mesenchymal reverting transition (EMrT) in nasopharyngeal carcinoma (NPC). Protein expression levels of p-Akt (Ser473), and the epithelial-to-mesenchymal transition (EMT) markers E-cadherin, vimentin, $\alpha$ smooth muscle actin ( $\alpha$-SMA), were examined in clinical samples from 130 cases of undifferentiated nonkeratinizing NPC, and 20 cases of benign nasopharyngitis. The relationship between protein expression levels and the statue of NPC lymph node metastasis was analyzed. The poorly-differentiated NPC cell line CNE2Z was treated with various concentrations of the PI3K inhibitor, LY294002, and western blotting and quantitative polymerase chain reaction assays were used to analyze the activation of PI3K/Akt signaling and expression of E-cadherin, vimentin and $\alpha$-SMA. The ability of cellular migration and invasion was assessed using Transwell assays. The in vivo effects of LY294002 on metastasis and expression of EMT markers in CNE2Z cells was evaluated using tumor xenograft experiments. The expression levels of p-Akt (Ser473) in NPC samples were higher than those in nasopharyngitis. There were reduced levels of membrane E-cadherin protein expression, and increased cytosol vimentin and $\alpha$-SMA expression levels in NPC samples compared with those in nasopharyngitis samples. High expression levels of p-Akt (Ser473), vimentin, and $\alpha$-SMA, and low expression levels of E-cadherin were positively associated with lymph node metastasis of NPC cells. Treating CNE2Z
\end{abstract}

Correspondence to: Dr Hanguo Jiang or Dr Wei Jie, Department of Pathology, School of Basic Medicine Science, Guangdong Medical College, Zhanjiang, Guangdong 524023, P.R. China

E-mail: jianghg12@163.com

E-mail: jiewei74@126.com

Key words: nasopharyngeal carcinoma, PI3K/Akt signaling, mesenchymal-epithelial transition, mesenchymal-epithelial reverting transition, LY294002 cells with LY294002 inhibited p-Akt (Ser473), vimentin and $\alpha$-SMA expression but upregulated E-cadherin expression, leading to significantly attenuated cell invasion and migration. Administration of mice with LY294002 resulted in upregulation of membrane E-cadherin, and downregulation of vimentin and $\alpha$-SMA in CNE2Z xenografts, with reduced pulmonary metastasis. Our findings suggest that inhibiting the PI3K/ Akt pathway using LY294002 attenuated NPC metastasis via induction of EMrT.

\section{Introduction}

Nasopharyngeal carcinoma (NPC) is one of the most common carcinomas in Southern China, particularly in Guangdong Province; however, it is rare in most other regions of the world $(1,2)$. NPC cells are highly malignant, often invading adjacent regions and metastasizing to regional lymph nodes and distant organs. Despite radiation therapy or adjunctive chemotherapy, 30-40\% of NPC patients die from local recurrence and distant metastasis (3). A better understanding of the molecular mechanisms contributing to NPC progression may provide new insights into possible therapeutic targets.

Increasing evidence indicates that metastasis of many epithelium-derived tumors is closely correlated with epithelialto-mesenchymal transition (EMT) (4-7). EMT is characterized by cells losing their epithelial morphology, and acquiring fibroblast-like properties. They also exhibit reduced intercellular adhesion and increased motility (8). Loss of E-cadherin expression and increased expression levels of mesenchymal cell markers such as vimentin, $\mathrm{N}$-cadherin, fibronectin and $\alpha$ smooth muscle actin ( $\alpha$-SMA) appear to be strongly correlated with EMT. Suppression of E-cadherin by transcriptional regulators such as Snail or Twist has emerged as a critical step driving EMT (9). Previously, it was reported that cancer cells could acquire epithelial-to-mesenchymal reverting transition (EMrT) to adapt to metastatic microenvironments, with re-expression of E-cadherin, an indicator of EMrT $(10,11)$. Under such conditions, cancer cells continue to display phenotypic plasticity beyond the EMT that initiates metastasis. To date, little is known about the precise mechanisms, biology, and clinical significance of EMrT in NPC. 
Previous studies revealed that several signaling pathways are involved in EMT and EMrT $(9,12,13)$. The phosphatidylinositol-3 OH kinase/protein kinase B (PI3K/Akt) signaling pathway is constitutively activated in various epithelial cancers, and plays an important role in tumor formation and metastasis (14,15). Phosphatidylinositol 3,4,5-triphosphate (PIP3), generated from PI3K, acts as a lipid second messenger essential for the translocation of Akt to the plasma membrane. Akt is phosphorylated at two sites, T308 in the kinase domain and S473 in the regulatory tail. Phosphorylation at T308 and S473 is essential for maximal Akt activation. Phosphorylated Akt (p-Akt) regulates the function of a broad array of intracellular proteins involved in fundamental processes, including cell proliferation, cell death, cell motility/adhesion, cell transformation, neovascularization and the inhibition of apoptosis. It has been reported that Akt suppresses transcription of the E-cadherin gene $(10,16)$. This transcriptional suppression induces responses leading to the conversion of epithelial cells into invasive mesenchymal cells.

A chemical inhibitor of PI3K, 2-4-morpholinyl-8-phenylchromone (LY294002), has been extensively used to study the role of the PI3K/AKT pathway in normal and transformed cells. Inactivation of PI3K using LY294002 has been shown to lead to the dephosphorylation of Akt at T308 and S473. A consequence of this is induction of G1 arrest during cell growth, which leads to apoptosis. Inhibitors of PI3K also possess antitumor activity in vitro and in vivo in a variety of tumors. It is possible that cells constitutively expressing active Akt become dependent on its ability to promote survival. Although these results have been observed in many human cancers, the effects of LY294002 on EMrT and metastasis in human NPC remain unclear. In the present study, we investigated the role of PI3K/Akt signaling activation and inhibition in EMrT for human NPC cases.

\section{Materials and methods}

Sample collection and ethics. Human benign nasopharyngeal samples (chronic nasopharyngitis, $n=20$ ) and NPC samples $(n=130)$ were obtained from the Affiliated Gaozhou Hospital, and the Affiliated Hospital of Guangdong Medical College. All NPC patients were diagnosed with non-keratinizing carcinoma following histological examination. Tissues were paraffin-embedded and sectioned (4- $\mu \mathrm{m}$ thickness). Of the 130 NPC cases, 53 involved local lymph node metastasis; of these 53 cases, 23 were paired with primary and metastatic lesions. The use of human tissues in this study was approved by the Ethics Council of the Affiliated Gaozhou Hospital, and the Affiliated Hospital of Guangdong Medical College.

Immunohistochemistry (IHC). Paraffin-embedded sections were used for immunohistochemical analysis of $\mathrm{p}-\mathrm{Akt}$ (Ser473), E-cadherin, vimentin and $\alpha$-SMA expression. Sections were deparaffinized and rehydrated, then heatinduced antigen retrieval at $95^{\circ} \mathrm{C}$ was conducted in sodium citrate buffer (10 mM, pH 6.0). Endogenous peroxidases were blocked using $0.3 \%(\mathrm{v} / \mathrm{v}) \mathrm{H}_{2} \mathrm{O}_{2}$, while binding of nonspecific proteins was blocked with $10 \%$ goat serum. Sections were then incubated with primary antibodies against p-Akt (Ser473) (1/200 dilution; Cell Signaling, Danvers, MA, USA),
E-cadherin (1/200) and vimentin (1/200) (both from Cell Signaling), and $\alpha$-SMA (1/100; Maixin Bio, Fujiang, China) at $4^{\circ} \mathrm{C}$ overnight. Non-immune immunoglobulin $\mathrm{G}(\mathrm{IgG})$ was used as a negative control. Antigenic sites were visualized using a streptavidin-peroxidase (SP) and a 3,3'-diaminobenzidine (DAB) kit (ZSGB-BIO, Beijing, China). Expression of p-Akt (Ser473), vimentin, and $\alpha$-SMA was localized to the cytoplasm, while E-cadherin signals were observed in the cell membrane or cytoplasm. Scoring of sections was conducted by two experienced pathologists, blinded to the identity of samples. Staining intensity was classified as 0 (negative); 1 (weak); 2 (moderate); and 3 (strong). The proportion of p-Akt (Ser473)-, E-cadherin-, vimentin- and $\alpha$-SMA-positive cells was scored as 1 (0-9\% positive); 2 (10-50\%); and 3 (>50\%). Samples with a sum immunoreactive score (IRS) $\geq 1$ were considered to be positive for p-Akt (Ser473), E-cadherin, vimentin and $\alpha$-SMA.

Cell culture. The poorly differentiated human NPC cell line, CNE2Z, was maintained as described previously (17-19). Briefly, CNE2Z cells were cultured in Dulbecco's modified Eagle's medium (DMEM; Life Technologies, Guangzhou, China) supplemented with $10 \%$ fetal bovine serum (FBS; Gibco), $100 \mathrm{U} / \mathrm{ml}$ penicillin and $100 \mu \mathrm{g} / \mathrm{ml}$ streptomycin at $37^{\circ} \mathrm{C} / 5 \% \mathrm{CO}_{2}$. LY294002 (Cayman Chemical Co., Ann Arbor, MI, USA) was dissolved in dimethyl sulfoxide (DMSO), and the final concentration of DMSO in growth medium was $0.5 \%$ (v/v). CNE2Z cells were incubated in DMEM containing $0.5 \%$ FBS for $24 \mathrm{~h}$; media were then replaced with complete growth medium containing LY294002 at $10,25,50$ or $75 \mu \mathrm{M}$, or DMSO $(0.5 \%, \mathrm{v} / \mathrm{v})$ for $48 \mathrm{~h}$; cells were then harvested for use in relevant experiments.

In vitro cell invasion and migration assays. Cell invasion assays were conducted using Transwell chambers containing membranes with $8 \mu \mathrm{m}$ pores. The upper surfaces of the Transwell chambers were coated with Matrigel matrix $(250 \mu \mathrm{l} / \mathrm{ml})$ overnight at $4^{\circ} \mathrm{C}$, then rehydrated with $0.1 \%(\mathrm{w} / \mathrm{v})$ bovine serum albumin (BSA) in DMEM for $1 \mathrm{~h}$ at $37^{\circ} \mathrm{C}$. Transwell chambers were then placed into 24-well culture plates. CNE2Z cells $\left(5 \times 10^{5}\right)$ were suspended in $200 \mu$ l of complete growth media supplemented with LY294002 $(10,25,50$ or $75 \mu \mathrm{M})$, or DMSO $(0.5 \%, \mathrm{v} / \mathrm{v})$, and were then added to the upper chambers and allowed to migrate toward the underside of the membrane. Membranes were fixed with $3.5 \%$ paraformaldehyde $24 \mathrm{~h}$ later; cells on the upper surface of the membrane were removed by wiping with a cotton swab, and then membranes were mounted onto glass slides. Cells on the lower faces of the membranes were counted, with 20 random fields of view per membrane counted for each assay. For cell migration assays, the protocol was similar to that used in cell invasion assays, except that Matrigel was not added to the upper chambers.

Quantitative polymerase chain reaction ( $q P C R)$ assays. The CNE2Z cells were treated with LY294002 (10, 25, 50 and $75 \mu \mathrm{M})$ and DMSO $(0.5 \%, \mathrm{v} / \mathrm{v})$ for $24 \mathrm{~h}$. Total RNA was extracted using Takara RNAiso Plus reagent (Takara Biotechnology Co., Ltd., China). We used the Promega RT System and oligo(dT)18, along with total RNA $(1 \mu \mathrm{g})$ as a template to generate first strand cDNA. Specific primer pairs 
Table I. Expression of p-Akt (Ser473), membrane E-cadherin, cytosol vimentin and $\alpha$-SMA in clinical nasopharyngitis and NPC samples.

\begin{tabular}{|c|c|c|c|c|c|c|c|c|c|}
\hline Conditions & $\mathrm{n}$ & $\begin{array}{c}\text { p-Akt (Ser473) } \\
+(\%)\end{array}$ & P-value & $\begin{array}{l}\text { E-cadherin } \\
+(\%)\end{array}$ & P-value & $\begin{array}{l}\text { Vimentin } \\
+(\%)\end{array}$ & P-value & $\begin{array}{l}\alpha \text {-SMA } \\
+(\%)\end{array}$ & P-value \\
\hline Tissue type & & & $<0.001$ & & $<0.05$ & & $<0.001$ & & $<0.01$ \\
\hline NPC & 130 & 99 (76.2) & & $98(75.4)$ & & $53(40.8)$ & & 48 (36.9) & \\
\hline Nasopharyngitis & 20 & $5(25.0)$ & & $20(100.0)$ & & 0 & & 0 & \\
\hline Metastasis & & & $<0.01$ & & $<0.01$ & & $<0.001$ & & $<0.05$ \\
\hline With & 53 & 47 (88.7) & & $33(62.3)$ & & $32(60.4)$ & & $25(47.2)$ & \\
\hline Without & 77 & $52(67.5)$ & & $65(84.4)$ & & $21(27.3)$ & & $23(29.9)$ & \\
\hline Matched lesion & & & $<0.05$ & & $<0.05$ & & $<0.05$ & & $>0.05$ \\
\hline Primary & 23 & $10(43.5)$ & & $18(78.3)$ & & $15(65.2)$ & & $7(30.4)$ & \\
\hline Secondary & 23 & $18(78.3)$ & & $11(47.8)$ & & $7(30.4)$ & & $13(56.5)$ & \\
\hline
\end{tabular}

$\alpha$-SMA, $\alpha$ smooth muscle actin; NPC, nasopharyngeal carcinoma.

for the amplification of E-cadherin (5'-TTG CTA CTG GAA CAG GGA CAC-3' and 5'-CCC GTG TGT TAG TTC TGC TGT-3'), vimentin (5'-TGC GTG AAA TGG AAG AGA ACT-3' and 5'-TCA GGT TCA GGG AGG AAA AGT-3'), $\alpha$-SMA (5'-TCC CTT GAG AAG AGT TAC GAG TTG-3' and 5'-ATG ATG CTG TTG TAG GTG GTT TC-3'), and $\beta$-actin (5'-TGA CGT GGA CAT CCG CAA AG-3' and 5'-CTG GAA GGT GGA CAG CGA GG-3') were synthesized by Sangon Biotech Co., Ltd. (Shanghai, China). The total reaction $(20 \mu \mathrm{l})$ comprised $10 \mu \mathrm{l}$ of SYBR-Green I PCR Master Mix (Roche), $0.8 \mu \mathrm{l}$ of each primer $(10 \mu \mathrm{M}), 2 \mu \mathrm{l}$ of cDNA and $6.4 \mu 1$ of double-distilled water. Thermal cycling involved an initial denaturation step at $95^{\circ} \mathrm{C}$ for $5 \mathrm{~min}$, followed by 45 cycles at $95^{\circ} \mathrm{C}$ for $25 \mathrm{sec}$, and $60^{\circ} \mathrm{C}$ for $60 \mathrm{sec}$, in a LightCycler $480 \mathrm{II}$ instrument (Roche China Ltd., Shanghai, China). The relative abundances of target mRNAs were determined from $\mathrm{Ct}$ values and plotted as fold-change compared with the control group. The transcription levels of $\beta$-actin served as a loading control.

Western blot analysis. Cells were homogenized with lysis buffer (1\% Triton X-100, $50 \mathrm{mM}$ Tris- $\mathrm{HCl} \mathrm{pH} 7.5,0.1 \%$ SDS, $150 \mathrm{mM} \mathrm{NaCl}, 10 \%$ glycerol, $1.5 \mathrm{mM} \mathrm{MgCl}{ }_{2}, 1 \mathrm{mM}$ PMSF, $0.1 \mathrm{mM} \mathrm{NaVO}, 0.1 \mathrm{mM}$ benzamidine, $5 \mu \mathrm{l} / \mathrm{ml}$ leupeptin and $5 \mu \mathrm{l} / \mathrm{ml}$ aprotinin), and protein homogenates were separated by sodium dodecyl sulfate-polyacrylamide gel electrophoresis (SDS-PAGE), and then transferred to nitrocellulose membranes. Membranes were incubated with primary antibodies against p-Akt (Ser473) (1:1,000 dilution), E-cadherin (1:500), vimentin (1:500) (all from Cell Signaling), $\alpha$-SMA (1:500; Maxin Bio), and $\beta$-actin (1:1000; Santa Cruz, Dallas, TX, USA) at $4^{\circ} \mathrm{C}$ overnight. Membranes were washed twice and incubated with horseradish peroxidase-conjugated secondary antibodies for $2 \mathrm{~h}$ at room temperature. Protein bands were visualized using enhanced chemiluminescent reagents (Thermo Fisher, Rockford, IL, USA) and analyzed using an InGenius LHR Gel Documentation System (Syngene, Frederick, MD, USA).

Tumor xenograft experiments. Specific pathogen-free (SPF) Balb/c null mice (6-8 weeks old) were purchased from the
Experimental Animal Center of Guangdong Medical College. Mice were housed in a facility with controlled temperature, humidity, and a $12 \mathrm{~h}$ light/dark cycle. All animal procedures were conducted in accordance with protocols approved by the Institutional Animal Care and Use Committee (IACUC) of Guangdong Medical College. CNE2Z cells (1x10 6 cells/mouse) were subcutaneously injected $(200 \mu \mathrm{l})$ into the right flanks of mice. After 1 week, tumor-bearing mice were intraperitoneally injected twice a week with $0.5 \%$ DMSO $(n=4)$ or LY294002 $(10,25,50$ or $75 \mathrm{mg} / \mathrm{kg}$; $\mathrm{n}=4$ for each group) for 4 weeks. Mice were monitored, with body weight and tumor sizes measured twice a week. All mice were euthanized at the end of the treatment period. Portions of the xenografts were fixed in natural formalin, and embedded in paraffin for immunohistochemical analysis of EMT markers. Xenografts that were not fixed were stored at $-80^{\circ} \mathrm{C}$. Lungs of each mouse were collected, the intermittent sections (10 sections/lung, 20 sections/mouse) were stained with $H \& E$ and the tumor metastatic lesions were calculated.

Statistical analysis. Statistical analyses were conducted using GraphPad Prism (GraphPad Software, CA, USA). We used $\chi^{2}$ tests to analyze and compare expression of p-Akt (Ser473) and EMT markers in clinical samples. Data for in vitro experiments are expressed as the means \pm SD. Differences between two groups were analyzed using Student's t-test. For comparison of multiple groups, ANOVAs were used followed by the Student-Newman-Keuls test. P-values $<0.05$ were considered to indicate a statistically significant difference.

\section{Results}

Activation of PI3K/Akt signaling and expression of EMT markers in NPC. Positive expression of p-Akt (Ser473) in chronic nasopharyngitis and NPC samples was observed in $25 \%$ $(5 / 20)$ and $76.2 \%(99 / 130)$ of samples, respectively $(\mathrm{P}<0.001)$ (Table I, Fig. 1), indicating the activation of PI3K/Akt signaling in NPCs. Expression levels of membrane E-cadherin, cytosol vimentin and $\alpha$-SMA proteins in chronic nasopharyngitis 


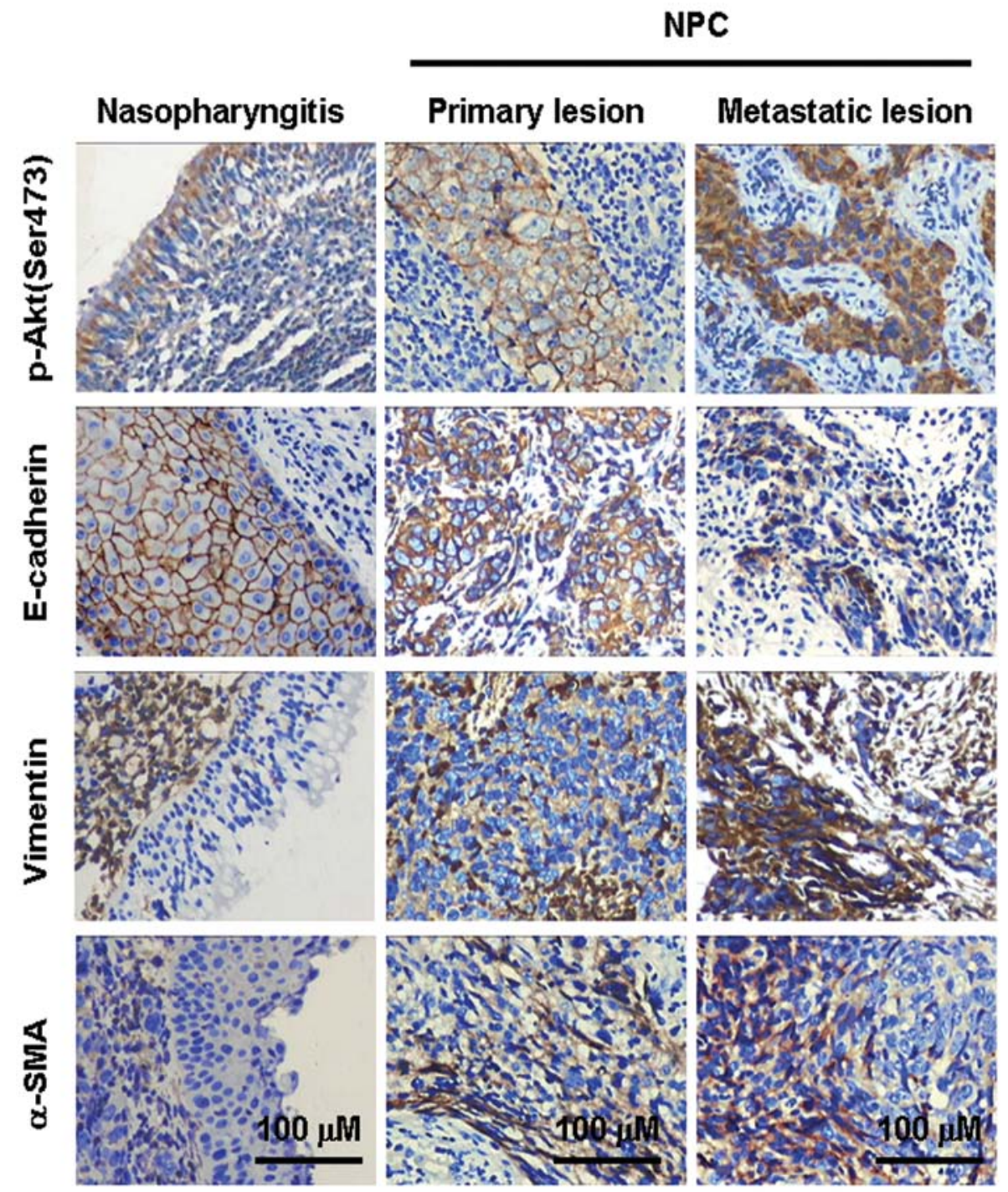

Figure 1. Immunohistochemical analysis of p-Akt (Ser473) and EMT marker expression in clinical samples from nasopharyngitis and nasopharyngeal carcinoma (NPC). Non-immune IgG was used as a negative control. E-cadherin expression in epithelial cells of nasopharyngitis samples was located in membranes while it was mainly located in the cytoplasm of metastatic NPC cells. Spindle and fibroblast-like tumor cells in NPC metastatic samples showed intense staining for vimentin and $\alpha$-SMA. Left, nasopharyngitis; middle, NPC primary lesions; right, NPC metastatic lesions. Magnification, $\mathrm{x} 200$. Scale bar, $100 \mu \mathrm{M}$.

samples were $100 \%(20 / 20), 0 \%$ and $0 \%$, respectively. For NPC samples, expression levels of these proteins were $75 \%$ $(98 / 130), 41 \%(53 / 130)$ and $37 \%(48 / 130)(\mathrm{P}<0.05,0.001$ and 0.01 , respectively). Spindle and fibroblast-like tumor cells in NPC samples exhibited intense vimentin and $\alpha$-SMA staining. These results indicated that, when compared with the benign chronic nasopharyngitis, the NPCs presented a much stronger activation of PI3K/Akt signaling, obvious downregulation in membrane E-cadherin and upregulation in cytosol vimentin and $\alpha$-SMA protein expressions.

Activation of PI3K/Akt signaling and EMT contribute to clinical NPC metastasis. Of the 130 NPC samples, 53 cases had signs of cervical lymph node metastasis. High levels of p-Akt (Ser473) expression were observed in cases with local lymph node metastasis compared with those without metastasis [89\% (47/53) vs. 68\% (52/77), $\mathrm{P}<0.01]$. Expression levels of membrane E-cadherin, cytosol vimentin and $\alpha$-SMA in NPC samples with local lymph node metastasis were $62 \%$
(33/53), 60\% (32/53) and 47\% (25/53), and 84\% (65/77), 27\% $(21 / 77)$ and $30 \%(23 / 77)$ in samples without local lymph node metastasis $(\mathrm{P}<0.01,0.001$ and 0.05 , respectively). To further investigate the role of PI3K/Akt activation and EMT occurrence in NPC metastasis, we analyzed the expression of p-Akt (Ser473) and EMT genes in NPC samples matched with primary and secondary lesions $(\mathrm{n}=23)$. Although expression of $\alpha$-SMA was not significantly different between primary and metastatic lesions, higher p-Akt (Ser473) and vimentin expression levels, and lower membrane E-cadherin expression levels, were observed in secondary NPC metastatic lesions $(\mathrm{P}<0.05)$ (Table I).

Inhibition of PI3K/Akt signaling alters expression of $p-A k t$ (Ser473) and EMT marker genes in CNE2Z cells in vitro. Treatment of CNE2Z cells with LY294002 significantly suppressed p-Akt (Ser473) expression in a concentrationdependent manner; particularly at $75 \mu \mathrm{M}$, the expression of p-Akt (Ser473) was almost completely attenuated (Fig. 2A). 


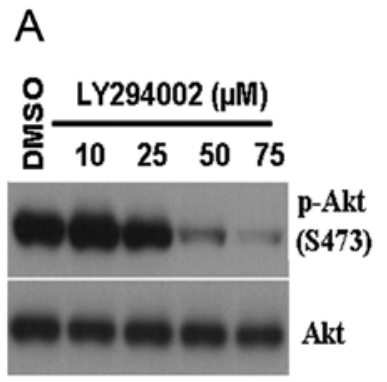

B

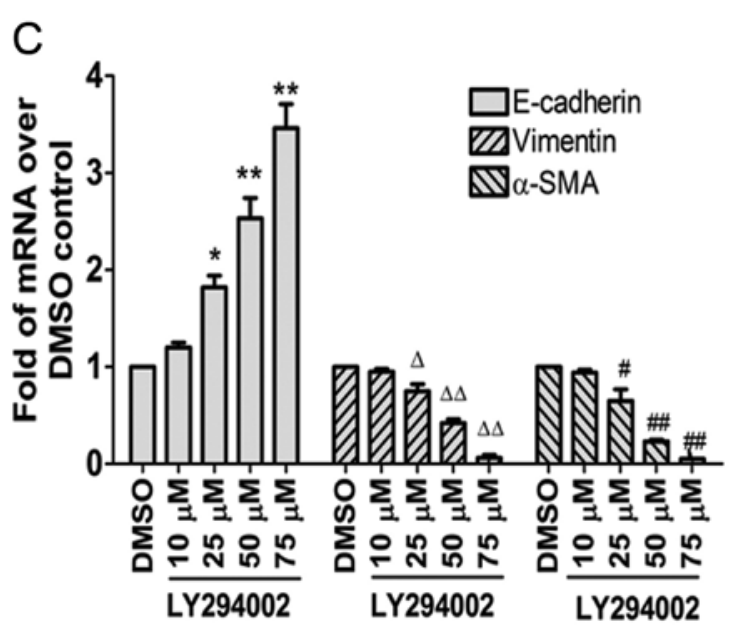

Figure 2. Activation of PI3K/Akt signaling and expression of E-cadherin, vimentin and $\alpha$-SMA in CNE2Z cells. (A) p-Akt (Ser473) and total Akt expression in CNE2Z cells treated with LY294002 or vehicle (DMSO) were examined by western blotting. (B) Expression of E-cadherin, vimentin and $\alpha$-SMA in CNE2Z cells treated with LY294002 or vehicle (DMSO) were examined by western blotting. (C) qPCR analysis of LY294002-treated CNE2Z cells. $\beta$-actin served as the loading control. ${ }^{*, \Delta, *} \mathrm{P}<0.05$ vs. DMSO; ${ }^{* *, \Delta \triangle, \# \#} \mathrm{P}<0.01$ vs. DMSO.

Treatment of CNE2Z cells with LY294002 led to upregulation of E-cadherin and downregulation of vimentin and $\alpha$-SMA at the protein and mRNA levels (Fig. 2B and C), and to EMrT in vitro.

Inhibition of PI3K/Akt signaling attenuates invasion and migration in CNE2Z cells in vitro. The ability of cellular migration and invasion was used to assess functional changes in vitro following inhibition of PI3K/Akt signaling. As we expected, LY294002 treatment of CNE2Z cells indeed significantly suppressed cell migration and invasion in a concentration-dependent manner (Fig. 3).

Effects of LY294002 on the expression of EMT marker genes in vivo. Western blot results revealed that xenografts from mice administered with DMSO displayed low levels of E-cadherin expression and high levels of vimentin and $\alpha$-SMA expression. Mice treated with LY294002 had increased levels of E-cadherin, and decreased levels of vimentin and $\alpha$-SMA that were dependent on the concentration of LY294002 (Fig. 4). Consistent with the western blot results, the IHC results exhibited the same tendencies (Fig. 5), and these results were consistent with those found in vitro (Fig. 2).

Effects of LY294002 on NPC cells in pulmonary metastasis in vivo. After all mice were euthanized, metastatic lesions in
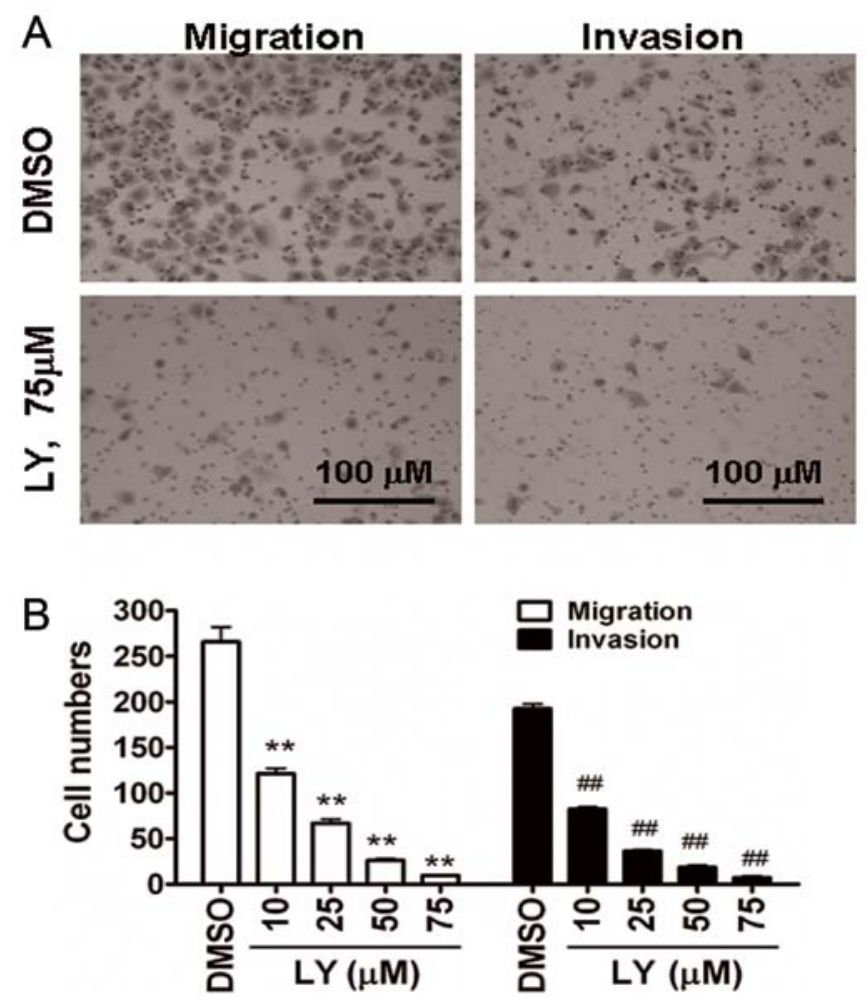

Figure 3. Treatment of CNE2Z cells with LY294002 suppresses cell migration and invasion. (A) Representative images of cell migration and invasion. (B) Numbers of migrating and invading CNE2Z cells. ${ }^{* *}$,\#\# $\mathrm{P}<0.01$ vs. DMSO. Scale bar, $100 \mu \mathrm{M}$.

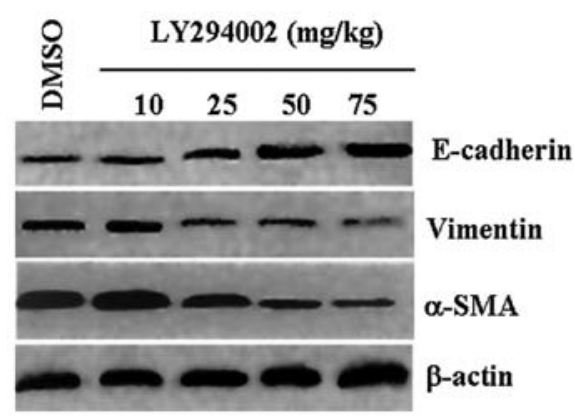

Figure 4. Expression of E-cadherin, vimentin and $\alpha$-SMA in xenografts of CNE2Z cell-bearing mice. Western blot analysis of EMT marker expression, with $\beta$-actin serving as loading control.

organs such as the liver, spleen, lung and brain were examined. At the gross level, metastatic lesions were not found in the liver, spleen, lung and brain of any mice. At the microscopic level, pulmonary metastatic lesions were observed in each group, with the following mean total number of pulmonary metastatic lesions: $23.25,11.25,3.75,0$ and 0 , respectively $(\mathrm{P}<0.001$, Fig. 6).

\section{Discussion}

The PI3K/Akt cascade is known to be an important signal transduction pathway involved in cell survival and apoptosis. This pathway is activated by other factors that play a critical role in 


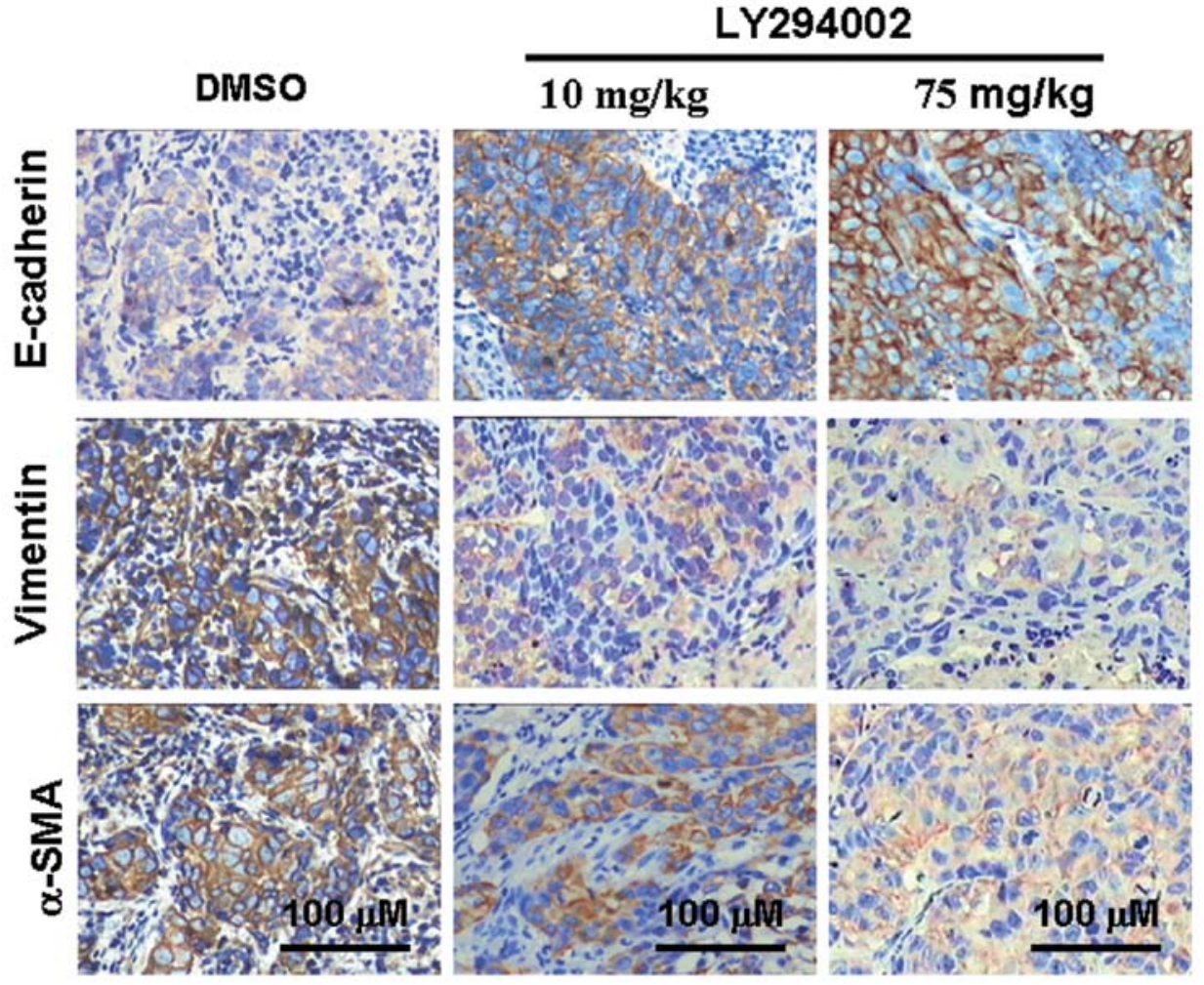

Figure 5. Immunohistochemical analysis of E-cadherin, vimentin and $\alpha$-SMA expression in xenografts of CNE2Z cell-bearing mice. Primary tumor masses were used for immunohistochemical analysis of EMT marker expression. Left, DMSO; middle, $10 \mathrm{mg} / \mathrm{kg} \mathrm{LY294002;} \mathrm{right,} 75 \mathrm{mg} / \mathrm{kg} \mathrm{LY294002.}$ Magnification, x200. Scale bar, $100 \mu \mathrm{M}$.

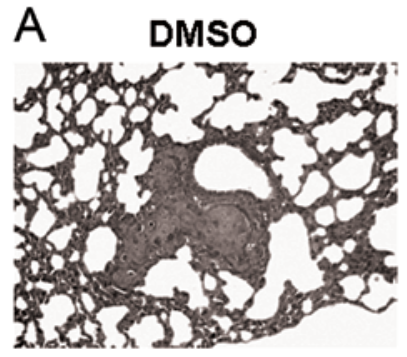

LY294002, $10 \mathrm{mg} / \mathrm{kg}$

B

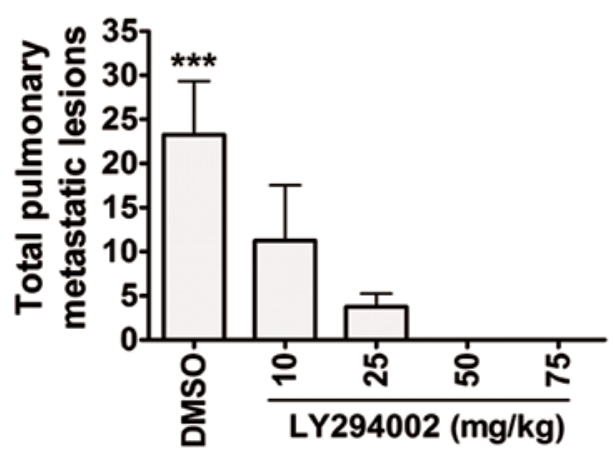

Figure 6. Pulmonary metastasis of CNE2Z cell-bearing mice. (A) Pulmonary metastatic lesions stained with hematoxylin and eosin. Magnification, x200. Scale bar, $100 \mu \mathrm{M}$. (B) Total pulmonary tumor metastatic lesions in CEN2Z cell-bearing mice. ${ }^{* * *} \mathrm{P}<0.001$ vs. other groups.

regulating tumor cell growth, survival, differentiation, motility and invasion (14). We have previously shown that inhibition of PI3K/Akt signaling using LY294002 inhibits CNE2Z cell proliferation, and induces apoptosis in vitro and in vivo (20). As a hallmark of malignant cancers, invasion and metastasis are major features that contribute to cancerous progression (21). Previous findings have indicated that metastasis of many epithelium-derived tumors have a close correlation with EMT. During EMT, epithelial cell-derived tumor cells acquire fibroblast-like properties. Loss of the E-cadherin protein is a key molecular event during EMT $(10,22)$.

In the present study, we examined the expression of p-Akt (Ser473) and EMT markers in chronic nasopharyngitis and NPC tissues. Our findings indicated that higher levels of p-Akt (Ser473), vimentin and $\alpha$-SMA expression, and lower membrane E-cadherin expression levels were observed in clinical NPC samples, indicating that activation of PI3K/Akt signaling and the occurrence of EMT was associated with NPC pathogenesis. We then assessed the relationship between changes in p-Akt (Ser473) and EMT marker expression levels to NPC metastasis. Consistent with our expectations, activation of PI3K/Akt signaling and occurrence of EMT were associated with NPC metastasis. Results from the 23 cases of NPC patients matched with primary and secondary lesions further highlighted this relationship. We observed expression of E-cadherin in the membranes of benign nasopharyngeal epithelia, while decreased levels of membrane expression and increased cytoplasmic E-cadherin expression were apparent in NPC tumor cells. Vimentin and $\alpha$-SMA expression was lacking in benign nasopharyngeal epithelial cells, except for some stromal cells. Certain NPC cells, particularly spindle and fibroblast-like tumor cells, displayed increased levels of vimentin and $\alpha$-SMA expression; however, these were reduced 
in adjacent epithelial-like cancer cells. Our results confirmed that EMT was detected in clinical NPC samples. The occurrence of EMT has a close relationship with metastasis of NPC cells, as detailed in other reports (23-25).

Treatment of the CEN2Z cell line with LY294002 significantly attenuated the levels of p-Akt(ser473)/Akt in a concentration-dependent manner, indicating a suppression of PI3K/Akt signaling in these cells. Increased levels of E-cadherin, and lower levels of vimentin and $\alpha$-SMA expression, were observed. Consistent with our expectations, these molecular changes in LY294002-treated CNE2Z cells resulted in reduced migration and invasion of cells. Our findings in vitro showed that inhibition of PI3K/Akt signaling led to EMrT in NPC cells. Our results are supported by those from other research groups, which showed that inhibition of Akt led to upregulation of E-cadherin in lung (26) and gastric cancer (27) cells.

The microenvironment of cancer cells is very complex and flexible. Epithelial cell-derived cancer cells acquire features of stromal cells and re-express E-cadherin to adapt to their new environment for certain stimuli. This leads to EMrT and abrogated cell motility $(6,28)$. To assess the effects of LY294002 on NPC cell metastasis in vivo, CNE2Z xenograft experiments were conducted. After tumor-bearing Balb/c null mice were administered LY294002 at various concentrations, primary tumor masses were analyzed for activation of Akt and expression of EMT markers. We observed attenuated Akt activation, which was accompanied by upregulation of E-cadherin and downregulation of vimentin and $\alpha$-SMA. Thus, LY294002 administration in vivo effectively leads to EMrT. Consistent with changes in EMT markers in xenografts, significantly reduced pulmonary metastasis was also observed. Therefore, we concluded that LY294002 administration in vivo inhibits tumor cell metastasis via EMrT, which was mediated by the suppression of PI3K/Akt signaling.

It has been reported that EMT of many epithelium-derived tumors is regulated by multiple signal transduction pathways that form complex networks (29). Our findings suggest that Akt kinase was one of the major effectors of EMT signals downstream of PI3K in NPC cells. Previous studies have demonstrated that EMT generates cells with the properties of stem cells (30). Recent reports also indicate that EMT occurs in NPC cells due to the existence of stem cell-like cancerous cells (31-33). Future studies should focus on investigating the contribution of Akt mediation to the biology of cancer stem cell-like cells in NPC.

There were some limitations to the present study; upstream PI3K/Akt signaling and inhibition of PI3K/Akt signaling besides using LY294002 in NPC should be considered, and the number of cases within animal experiments should be greater. Considering that activation of PI3K/Akt signaling crosstalks with other cascades (34), the present investigation outlined the clinical significance of LY294002 in the treatment of NPC.

In conclusion, we showed that EMT characteristics were apparent in NPC tissues, and that administration of LY294002 in CNE2Z cells induced upregulation of E-cadherin and downregulation of $\alpha$-SMA and vimentin in vitro and in vivo. This led to impaired cell metastasis via the induction of EMrT. Our results highlight the possibility of targeting the PI3K/ Akt pathway as a therapeutic strategy for controlling NPC cell invasion and metastasis. We consider that this concept for NPC may then be applied to other carcinomas.

\section{Acknowledgements}

This study was supported by grants from the Research Program of Guangdong Medical College (B2011018, B2010013 and Z2013004), and the Guangdong Provincial Medical Research Foundation (A2013421).

\section{References}

1. Cao SM, Simons MJ and Qian CN: The prevalence and prevention of nasopharyngeal carcinoma in China. Chin J Cancer 30: 114-119, 2011.

2. Chang ET and Adami HO: The enigmatic epidemiology of nasopharyngeal carcinoma. Cancer Epidemiol Biomarkers Prev 15: 1765-1777, 2006

3. Zhang Y, Lin ZA, Pan JJ, et al: Concurrent control study of different radiotherapy for primary nasopharyngeal carcinoma: intensity-modulated radiotherapy versus conventional radiotherapy. Ai Zheng 28: 1143-1148, 2009 (In Chinese).

4. Wu Y and Zhou BP: New insights of epithelial-mesenchymal transition in cancer metastasis. Acta Biochim Biophys Sin 40: 643-650, 2008

5. Lee JM, Dedhar S, Kalluri R and Thompson EW: The epithelialmesenchymal transition: new insights in signaling, development, and disease. J Cell Biol 172: 973-981, 2006.

6. Gao D, Vahdat LT, Wong S, Chang JC and Mittal V: Microenvironmental regulation of epithelial-mesenchymal transitions in cancer. Cancer Res 72: 4883-4889, 2012.

7. Talbot LJ, Bhattacharya SD and Kuo PC: Epithelial-mesenchymal transition, the tumor microenvironment, and metastatic behavior of epithelial malignancies. Int J Biochem Mol Biol 3: 117-136, 2012.

8. Thiery JP: Epithelial-mesenchymal transitions in tumour progression. Nat Rev Cancer 2: 442-454, 2002.

9. Huber MA, Kraut N and Beug H: Molecular requirements for epithelial-mesenchymal transition during tumor progression. Curr Opin Cell Biol 17: 548-558, 2005.

10. Wells A, Yates C and Shepard CR: E-cadherin as an indicator of mesenchymal to epithelial reverting transitions during the metastatic seeding of disseminated carcinomas. Clin Exp Metastasis 25: 621-628, 2008.

11. Chao YL, Shepard CR and Wells A: Breast carcinoma cells re-express E-cadherin during mesenchymal to epithelial reverting transition. Mol Cancer 9: 179, 2010

12. Zheng $\mathrm{H}$ and Kang Y: Multilayer control of the EMT master regulators. Oncogene 33: 1755-1763, 2014.

13. Lee YJ and Han HJ: Troglitazone ameliorates high glucoseinduced EMT and dysfunction of SGLTs through PI3K/Akt, GSK-3 $\beta$, Snaill, and $\beta$-catenin in renal proximal tubule cells. Am J Physiol Renal Physiol 298: F1263-F1275, 2010.

14. Nicholson KM and Anderson NG: The protein kinase B/Akt signalling pathway in human malignancy. Cell Signal 14: 381-395, 2002.

15. Sheng S, Qiao M and Pardee AB: Metastasis and AKT activation. J Cell Physiol 218: 451-454, 2009.

16. Grille SJ, Bellacosa A, Upson J, et al: The protein kinase Akt induces epithelial mesenchymal transition and promotes enhanced motility and invasiveness of squamous cell carcinoma lines. Cancer Res 63: 2172-2178, 2003.

17. Shen Z, Zeng Y, Guo J, et al: Over-expression of the special AT rich sequence binding protein 1 (SATB1) promotes the progression of nasopharyngeal carcinoma: association with EBV LMP-1 expression. J Transl Med 11: 217, 2013.

18. Shen Z, Jiang $X$, Zeng $C$, et al: High expression of ubiquitinconjugating enzyme $2 \mathrm{C}$ (UBE2C) correlates with nasopharyngeal carcinoma progression. BMC Cancer 13: 192, 2013.

19. Jie W, He QY, Luo BT, et al: Inhibition of Pim-1 attenuates the proliferation and migration in nasopharyngeal carcinoma cells. Asian Pac J Trop Med 5: 645-650, 2012.

20. Jiang H, Fan D, Zhou G, Li X and Deng H: Phosphatidylinositol 3-kinase inhibitor (LY294002) induces apoptosis of human nasopharyngeal carcinoma in vitro and in vivo. J Exp Clin Cancer Res 29: 34, 2010. 
21. Hanahan D and Weinberg RA: Hallmarks of cancer: the next generation. Cell 144: 646-674, 2011.

22. Hong KO, Kim JH, Hong JS, et al: Inhibition of Akt activity induces the mesenchymal-to-epithelial reverting transition with restoring E-cadherin expression in KB and KOSCC-25B oral squamous cell carcinoma cells. J Exp Clin Cancer Res 28: 28, 2009.

23. Luo WR, Chen XY, Li SY, Wu AB and Yao KT: Neoplastic spindle cells in nasopharyngeal carcinoma show features of epithelial-mesenchymal transition. Histopathology 61: 113-122, 2012.

24. Li XJ, Peng LX, Shao JY, et al: As an independent unfavorable prognostic factor, IL-8 promotes metastasis of nasopharyngeal carcinoma through induction of epithelial-mesenchymal transition and activation of AKT signaling. Carcinogenesis 33: 1302-1309, 2012.

25. Horikawa T, Yang J, Kondo S, et al: Twist and epithelial-mesenchymal transition are induced by the EBV oncoprotein latent membrane protein 1 and are associated with metastatic nasopharyngeal carcinoma. Cancer Res 67: 1970-1978, 2007.

26. Chen XF, Zhang HJ, Wang HB, et al: Transforming growth factor- $\beta 1$ induces epithelial-to-mesenchymal transition in human lung cancer cells via PI3K/Akt and MEK/Erk1/2 signaling pathways. Mol Biol Rep 39: 3549-3556, 2012.

27. Kang MH, Kim JS, Seo JE, Oh SC and Yoo YA: BMP2 accelerates the motility and invasiveness of gastric cancer cells via activation of the phosphatidylinositol 3-kinase (PI3K)/Akt pathway. Exp Cell Res 316: 24-37, 2010.
28. Ding S, Zhang W, Xu Z, et al: Induction of an EMT-like transformation and MET in vitro. J Transl Med 11: 164, 2013.

29. Thiery JP and Sleeman JP: Complex networks orchestrate epithelial-mesenchymal transitions. Nat Rev Mol Cell Biol 7: 131-142, 2006.

30. Battula VL,Evans KW,Hollier BG, et al: Epithelial-mesenchymal transition-derived cells exhibit multilineage differentiation potential similar to mesenchymal stem cells. Stem Cells 28: 1435-1445, 2010.

31. Kong QL, Hu LJ, Cao JY, et al: Epstein-Barr virus-encoded LMP2A induces an epithelial-mesenchymal transition and increases the number of side population stem-like cancer cells in nasopharyngeal carcinoma. PLoS Pathog 6: e1000940, 2010.

32. Guo D, Xu BL, Zhang XH and Dong MM: Cancer stem-like side population cells in the human nasopharyngeal carcinoma cell line CNE-2 possess epithelial mesenchymal transition properties in association with metastasis. Oncol Rep 28: 241-247, 2012.

33. Lin $\mathrm{CH}$, Shen YA, Hung PH, Yu YB and Chen YJ: Epigallocathechin gallate, polyphenol present in green tea, inhibits stem-like characteristics and epithelial-mesenchymal transition in nasopharyngeal cancer cell lines. BMC Complement Altern Med 12: 201,2012.

34. Jazirehi AR, Wenn PB and Damavand M: Therapeutic implications of targeting the PI3Kinase/AKT/mTOR signaling module in melanoma therapy. Am J Cancer Res 2: 178-191, 2012. 